\title{
Many-Objective Portfolio Optimization of Interdependent Projects with 'a priori' Incorporation of Decision-Maker Preferences
}

\author{
Laura Cruz ${ }^{1}$, Eduardo Fernandez ${ }^{2}$, Claudia Gomez ${ }^{1}$, Gilberto Rivera $^{3, *}$ and Fatima Perez ${ }^{4}$ \\ ${ }^{1}$ Postgraduate \& Research Division, Madero Institute of Technology, 89440, Tamaulipas, Mexico \\ ${ }^{2}$ Faculty of Civil Engineering, Autonomous University of Sinaloa, 80040, Sinaloa, Mexico \\ ${ }^{3}$ Computer Science in the Graduate Division, Tijuana Institute of Technology, 22500, Baja California, Mexico \\ ${ }^{4}$ Department of Applied Economics (Mathematics), University of Malaga, 29071, Malaga, Spain
}

Received: 6 Jul. 2013, Revised: 8 Oct. 2013, Accepted: 9 Oct. 2013

Published online: 1 Jul. 2014

\begin{abstract}
Project portfolio selection is one of the most important problems faced by any organization. The decision process involves multiple conflicting criteria, and has been commonly addressed by implementing a two-phase procedure. The first step identifies the efficient solution set; the second step supports the decision maker in selecting only one portfolio solution from the efficient set. However, several recent studies show the advantages gained by optimizing towards a region of interest (according to the decision maker's preferences) instead of approximating the complete Pareto set. However, these works have not faced synergism and its variants, such as cannibalization and redundancy. In this paper we introduce a new approach called Non-Outranked Ant Colony Optimization, which optimizes interdependent project portfolios with a priori articulation of decision-maker preferences based on an outranking model. Several experimental tests show the advantages of our proposal over the two-phase approach, providing reasonable evidence of its potential for solving real-world high-scale problems with many objectives.
\end{abstract}

Keywords: portfolio selection, interdependent projects, multiobjective metaheuristic optimization, preference incorporation, multicriteria decision

\section{Introduction}

Portfolio problems are ubiquitous in business and government organizations. Usually, there are more good ideas for projects or programmes than there are resources (funds, capacity, time, etc.) to support them [1]. Manufacturing enterprises recognize that success depends on the selection of research and development (R\&D) project portfolios, expecting that these projects will permit them to develop new products that generate growing benefits. Local governments allocate public funds to projects and programmes that improve social and educational services. Environmental regulations and alternative policy measures attempt to mitigate the harmful consequences of human activity [2]. To fight poverty, governments in underdeveloped countries fund many helpful social programmes. Portfolio consequences are usually described by multiple attributes related to the $\begin{array}{llll}\text { organizational } & \text { strategy. } & \text { A }\end{array}$ $z(x)=\left\langle z_{1}(x), z_{2}(x), \ldots, z_{p}(x)\right\rangle$ is associated with the consequences of a portfolio $x$ considering $p$ criteria. This is a vector representation of the portfolio's impact. In the simplest case, $z(x)$ is obtained from the cumulative sum of the benefits of the selected projects, but under interacting project conditions, it is necessary to consider the contribution of interdependent project groups. Without loss of generality, we can assume that higher criterion values are preferred to lower values. The best portfolio is obtained by solving the following problem:

$$
\max _{x \in R_{F}}\left\{\left\langle z_{1}(x), z_{2}(x), \ldots, z_{p}(x)\right\rangle\right\},
$$

where $R_{F}$ is the space of feasible portfolios, and is usually determined by the available budget, and by constraints for the kind of projects, social roles and geographic zones. Solving Problem (1) means finding the best compromise solution according to the system of preferences and values of the Decision Maker (DM).

\footnotetext{
*Corresponding author e-mail: riveragil@gmail.com
} 
In the scientific literature, the problem expressed by (1) has received great interest in the management of $R \& D$ by manufacturing and industrial enterprises (e.g. $[3,4,5$, $6,7,8]$ ). Most of these approaches can also be applied in the public sector. Perhaps what best characterizes the portfolio problems in non-profit organizations are the emphasis on intangible criteria and, probably, a higher number of project proposals and objectives to optimize. Many-objective problems are frequent in project portfolio optimization. For example, in socially responsible organizations, the number of criteria used for capital investment may be about a dozen (see [9]). Even more objective functions should be considered in basic research project management (cf. [10]). A high number of project proposals can apply for public support in a simple call for projects. For instance, in 2012 the US state of Georgia had a list of over 1600 applicant projects at the State Department of Transportation alone [11,12,13,14], with many potential interdependencies. There should be a large set of Pareto-efficient solutions to (1). However, the DM has to select only one portfolio according to her/his preferences for the consequences expressed by $z(x)$.

The specificity of such project portfolio problems with many objectives has been scarcely approached by the scientific literature. This paper is a contribution in this sense. It is structured as follows. Section 2 summarizes the most-widely accepted optimization model of the portfolio problem. Section 3 briefly reviews proposals for incorporating DM preferences in multi-objective optimization metaheuristics, and on this background, the method by Fernandez et al. $[10,15]$ is detailed. Our proposal is presented in Section 4, followed by test examples and comparisons with other approaches (Section 5). Finally, some conclusions are discussed in Section 6.

\section{Description and formalization of the problem}

Here, we follow the proposal by Stummer and Heidemberger in [5] that was also addressed by Doerner et al. $[16,17]$ and Carazo et al. [18,19].

Let $X$ be the set of applicant projects competing for resources. A portfolio (a subset of $X$ ) is typically represented by a binary vector $x=\left\{x_{1}, x_{2}, \ldots, x_{N}\right\}$, where $N$ is the total of project proposals; the variables $x_{j}$ indicate whether the project $j$ is included in the portfolio $\left(x_{j}=1\right)$ or $\operatorname{not}\left(x_{j}=0\right)$.

Let us denote by $f(j)=\left\{f_{1}(j), f_{2}(j), \ldots, f_{p}(j)\right\}$ the benefits provided by the $j$ th project. The benefits provided by portfolio $x$ are expressed by Equation (2):

$$
z(x)=\left\{z_{1}(x), z_{2}(x), \ldots, z_{p}(x)\right\},
$$

where $z_{k}(x)$ is defined as

$$
z_{k}(x)=\sum_{j=1}^{N} x_{j} \cdot f_{k}(j)+\sum_{i=1}^{S} g_{i}(x) \cdot a_{i, k} .
$$

In Equation (3), the first term is the cumulative sum of the benefits from the selected projects to the $k$ th objective function. The second term is the sum of the synergetic interactions among the projects in the portfolio. $S$ is the number of interactions that impact the objectives. Let us assume that those interactions have been identified by the DM. Function $g_{i}(x)$ indicates if the $i$ th interaction occurs in the portfolio $x$. If $A_{i}=\left\{A_{i, 1}, A_{i, 2}, \ldots, A_{i, N}\right\}$ is a binary vector that indicates which projects are affected by the $i$ th interdependency $\left(A_{i, j}=1\right.$ represents that the $j$ th project is considered in the $i$ th objective interaction), $g_{i}(x)$ may be defined as

$$
g_{i}(x)=\left\{\begin{array}{l}
1 \text { if } m_{i} \leq \sum_{j=1}^{N}\left(x_{j} \cdot A_{i, j}\right) \leq M_{i} \\
0 \text { otherwise }
\end{array}\right.
$$

In Equation (4), $m_{i}$ and $M_{i}$ are respectively the minimum and maximum number of projects required for synergy $i$ to occur, thus gaining additional benefits.

In Equation (3), $a_{i, k}$ is the value added to the $k$ th objective when the $i$ th synergy is activated. The interaction has been particularly named cannibalization if $a_{i, k}$ is negative.

Suppose that there are $q$ categories of resources destined for supporting project proposals. Let $\left\{\mathbb{B}_{1}, \mathbb{B}_{2}, \ldots, \mathbb{B}_{q}\right\}$ be the set containing the quantity of available resources for each category (e.g. financial, human or technological resources), and let $c_{j, k}$ be the amount of the $k$ th resource requested by project $j$. Thus, the total of the $k$ th resource needed for implementing portfolio $x$, is expressed by Equation (5):

$$
c_{k}(x)=\sum_{j=1}^{N} x_{j} \cdot c_{j, k}+\sum_{i=1}^{R} h_{i}(x) \cdot b_{i, k} .
$$

The first term in Equation (5) is the sum of resources consumed by the projects in $x$, without considering resource interactions. The second term is the sum concerning interactions that affect costs and resources requested. $R$ is the number of these interdependencies, $h_{i}(x)$ is a binary function that indicates if the $i$ th resource interaction occurs, and $b_{i, k}$ is the change in the $k$ th cost produced by the $i$ th interaction. $h_{i}(x)$ is defined in Equation (6) similarly to $g_{i}(x)$, but considering $n_{i}$ and $N_{i}$ as limits for activating synergy. Equation (6) presents the definition of $h_{i}(x)$ :

$$
h_{i}(x)=\left\{\begin{array}{l}
1 \text { if } n_{i} \leq \sum_{j=1}^{N}\left(x_{j} \cdot C_{i, j}\right) \leq N_{i}, \\
0 \text { otherwise, }
\end{array}\right.
$$

where $C_{i}=\left\{C_{i, 1}, C_{i, 2}, \ldots, C_{i, N}\right\}$ is a binary vector that indicates which projects are affected by the $i$ th cost interdependency. 
Of course, Problem (1) is subject to the budgetary constraint:

$$
c_{k}(x) \leq \mathbb{B}_{k} \quad \forall k \in\{1,2, \ldots, q\} .
$$

Besides Equation (7), other strategic and logical constraints could be regarded. For example:

-Constraints to ensure equitable conditions for all competent areas of the organization. All applicant projects are grouped according to pre-established criteria. The organization determines limits in terms of number of supported projects (or quantities of allocated budget) for each group.

-Constraints to prevent the presence of mutually-excluding projects. Some projects (primarily because of their nature and organizational rules) cannot simultaneously receive support in the same portfolio decision process. These projects often receive the adjective 'redundant'.

We are not taking into account project scheduling, thus we are tackling the stationary version of the problem presented in $[16,19]$; for this reason, all the concerns related to schedule are not included in either Equations (2-7) or the above-mentioned constraints. Conditions of partial support have no special processing, but it is possible to include dummy projects that represent different versions of the same project. So, dummy projects are treated like redundant proposals, in the same sense as it is suggested in $[5,16,17,18,19]$.

\section{An outline of the state of the art}

\subsection{A brief outline and some criticisms of previous approaches}

Only non-dominated solutions to (1) can fulfil the conditions necessary for being considered the best portfolio. So most solution methods seek to generate the Pareto frontier, and later, by some interactive method, multicriteria procedure or heuristic, try to identify the best compromise. These approaches assume that the DM has the capacity to make valid judgments about the set of efficient points until the best compromise is reached. This way to identify the best solution is commonly referred to as a posteriori preferences modelling [20].

In [21], Ghasemzadeh et al. model preferences using a weighted-sum function. They approximate the Pareto frontier by changing the weights and solving the resultant model by $0-1$ programming. Stummer and Heidenberger in [5] include synergy and redundancy in selecting R\&D projects; their procedure consists of three phases: 1) filtering the proposals and retaining the most promising projects in order to reduce the set of projects to a 'manageable' size, 2) generating the efficient frontier of portfolios for the reduced set by an integer linear programming method, and 3) supporting the decision-making process, helping the DM to identify the best compromise by an interactive process.

However, most recent works show the advantages of multi-objective metaheuristic methods to approximate the Pareto set (e.g. [8, 19,22, 23, 24, 25, 26, 27, 28]). Doerner et al. in [17] combine Ant Colony Optimization (ACO) with 0-1 dynamic mathematical programming to initialize the algorithm with enhanced solutions. One of the most complete proposals was suggested by Carazo et al. [18, 19]; they model interactions among projects (in the same way as Stummer and Heidenberger in [5]) and temporal dependencies, enabling the allocation of resources not used in previous periods. By means of a Scatter Search, Carazo et al. [18] outperform SPEA2 [29] in the range of 25-60 projects considering up to six objective functions.

Compared with multi-objective optimization methods based on mathematical programming, metaheuristic approaches exhibit relevant advantages:

-they have the ability to deal with a set of solutions (called a population) at the same time, allowing for the efficient frontier to be approximated in a single algorithm run, and

-they are less sensitive to the mathematical properties of objective functions and problem constraints.

However, many researchers have argued that, when the number of objective functions increases, the selection of appropriate individuals for conducting the population towards the Pareto frontier becomes more difficult (e.g. $[30,31,32,33])$. According to [32], other important concerns are the so-called Dominance Resistant Solutions (e.g. [34]). They are not Pareto solutions, but they have near-optimal values in some objectives though with a poor value in at least one of the remaining objectives. These solutions can be hardly dominated in a population. Their number grows as the dimension of the objective space is increased.

In the presence of many objectives, there are other important concerns associated with the a posteriori articulation of preferences:

1.The visualization of the Pareto front in high-dimensional objective spaces is very cumbersome.

2.The number of Pareto optimal points grows exponentially, making it hard to obtain a representative sample of the non-dominated frontier.

3.According to the famous Miller's paper [35], the human mind is limited to handling a small number of information pieces simultaneously, thus being questionable the issue of identifying the best compromise solution when the DM should compare even a small subset of non-dominated solutions in problems with many objectives.

Most approaches from the field of Multi-Criteria Decision Analysis (MCDA) do not perform well on large decision problems. Incomparability, non-transitivity, cyclic preferences and dependence with respect to 
'irrelevant alternatives' make it difficult to reach a reliable final prescription.

In order to make the decision making phase easier, the DM would agree incorporate his/her multicriteria preferences into the search process. This preference information is used to guide the search towards the Region of Interest (RoI) [36], the privileged zone of the Pareto frontier that best matches the DM's preferences.

The DM's preference information can be expressed in different ways. According to Bechikh [37], the most commonly-used ways are the following:

1.Those in which importance factors (weights) are assigned by the DM to each objective function (e.g. $[38,39,40])$.

2.Those in which the DM makes pair-wise comparisons on a subset of the current population, in order to rank the sample's solutions (e.g. [41,42,43,44,45,46]).

3.Those in which pair-wise comparisons between pairs of objective functions are performed in order to rank the set of objective functions (e.g. [47,48,49]).

4.Those based on goals or aspiration levels to be achieved by each objective (reference point) (e.g. [36, $50,51,52,53,54])$.

5.Those in which the DM identifies acceptable trade-offs between objective functions (e.g. [55]).

6.Those in which the DM supplies the model's parameters to build a fuzzy outranking relation (e.g. $[15,56])$

7.The construction of a desirability function which is based on the assignment of some desirability thresholds (e.g. [57]).

In the field of project portfolio optimization, the model proposed in [10] has shown substantial benefits for tackling these problems. This model is briefly explained below.

\subsection{The best portfolio in the sense of Fernandez et al. [10]}

The proposal by Fernandez et al. [10,15] is based on the relational system of preferences described in [58] by Roy. A crucial model is the degree of credibility of the statement ' $x$ is at least as good as $y$ '. This is represented as $\sigma(x, y)$ and could be calculated using proven methods from the literature, such as ELECTRE [59] and PROMETHEE [60]. Considering the parameters $\lambda, \beta$, and $\epsilon(0 \leq \epsilon \leq \beta \leq \lambda$ and $\lambda>0.5)$, the proposal in [10,15] identifies one of the following relations for each pair of portfolios $(x, y)$ :

1.Strict preference: Denoted as $x \mathrm{P} y$, represents the situation when the DM significantly prefers $x$. It is defined as a disjunction of the conditions:
(a) $x$ dominates $y$.
(b) $\sigma(x, y) \geq \lambda \wedge \sigma(y, x)<0.5$.

$$
\begin{aligned}
& \text { (c) } \sigma(x, y) \geq \lambda \wedge[0.5 \leq \sigma(y, x)<\lambda] \wedge \\
& {[\sigma(x, y)-\sigma(y, x)] \geq \beta}
\end{aligned}
$$

2.Indifference: From the DM's perspective, the two alternatives have a high degree of equivalence, so he/she cannot state that one is preferred over the other. This relationship is denoted as $x \mathrm{I} y$. In terms of $\sigma(x, y)$ this is defined as the conjunction of:

(a) $\sigma(x, y) \geq \lambda \wedge \sigma(y, x) \geq \lambda$.

(b) $|\sigma(x, y)-\sigma(y, x)| \leq \epsilon$.

3. Weak preference: Represented as $x \mathrm{Q} y$, this models a state of doubt between $x \mathrm{P} y$ and $x \mathrm{I} y$. It can be defined as the conjunction of:

(a) $\sigma(x, y) \leq \lambda \wedge \sigma(x, y) \geq \sigma(y, x)$.

(b) $\neg x \mathrm{P} y \wedge \neg x \mathrm{I} y$.

4.Incomparability: From the point of view of the DM, there is high heterogeneity between the alternatives, so he/she cannot set a preference relation between them. This is denoted as $x \mathrm{R} y$, and is expressed in terms of $\sigma(x, y)$ as $x \mathrm{R} y \Rightarrow \sigma(x, y)<0.5 \wedge \sigma(y, x)<0.5$.

5.k-Preference: This represents a state of doubt between $x \mathrm{P} y$ and $x \mathrm{R} y$, and is denoted as $x \mathrm{~K} y .(x, y) \in \mathrm{K}$ if the following three conditions are true:
(a) $0.5 \leq \sigma(x, y) \leq \lambda$.
(b) $\sigma(y, x)<0.5$.
(c) $\sigma(x, y)-\sigma(y, x)>\frac{\beta}{2}$.

Indifference corresponds to the existence of clear and positive reasons that justify equivalence between the two options. Additionally, incomparability represents situations where the DM cannot, or does not want to, express a preference. Strict preference is associated with conditions in which the DM has clear and well-defined reasons justifying the choice of one alternative over the other. However, because the DM usually shows non-ideal behaviour, the weak preference and the $k$-preference also exist. These relations can be considered as 'weakened' ways of the strict preference.

From a set of feasible portfolios $O$, the preferential system defines the following sets:

$1 . S(O, x)=\{y \in O \mid y \mathrm{P} x\}$ is composed of the solutions that strictly outrank $x$.

2.NS $(O)=\{x \in O \mid S(O, x)=\emptyset\}$ is known as the non-strictly-outranked frontier.

3.W $(O, x)=\{y \in N S(O) \mid y \mathrm{Q} x \wedge y \mathrm{~K} x\}$ is composed of the non-strictly-outranked solutions that weakly outrank $x$.

4.NW $(O)=\{x \in O \mid W(O, x)=\emptyset\}$ is known as the non-weakly-outranked frontier.

Besides the weak outranking, the net flow score is another measure used in $[10,15]$ to identify the DM's preferences in the non-strictly-outranked frontier. It can be defined as:

$$
F_{n}(x)=\sum_{y \in N S(O) \backslash\{x\}}[\sigma(x, y)-\sigma(y, x)] .
$$

Since $F_{n}(x)>F_{n}(y)$ indicates a preference for $x$ over $y$, Fernandez et al. [15] define: 
1.F $(O, x)=\left\{y \in N S(O) \mid F_{n}(y)>F_{n}(x)\right\}$ to be the set of non-strictly-outranked solutions that are greater in net flow to $x$.

2. $N F(O)=\{x \in N S(O) \mid F(O, x)=\emptyset\}$ to be the net-flow non-outranked frontier.

Fernandez et al. [10] proved that the best portfolio compatible with the fuzzy outranking relation $\sigma$ should be a non-strictly outranked solution that is simultaneously a non-dominated solution to the problem:

$$
\min _{x \in O}\{\langle|S(O, x)|,|W(O, x)|,|F(O, x)|\rangle\}
$$

As a consequence of the last remark, the best portfolio can be found through a lexicographic search, with pre-emptive priority favouring $|S(O, x)|$.

The above three-objective problem is a map of the original problem in (1). When the DM is confident on the preference model, he/she should accept that the best compromise is a non-dominated solution of Problem (9). It is also interesting that the equivalence between the problem in (1) and its mapped three-objective problem is valid independently of the original objective space dimension. This may be very important in solving portfolio problems with many objective functions [15].

The model parameters need to be adjusted according to the specific characteristics of the problem and of the DM. This can be done by an interaction between the DM and a Decision Analyst (DA), utilizing, if necessary, indirect elicitation methods to support this task [61,62, 63]. The DM should assess the parameters included in:

-the calculation of $\sigma$ (e.g. criterion weights and thresholds), and

-the system of preferences $(\lambda, \beta$ and $\epsilon)$.

This is not an easy task since DMs usually have difficulties in specifying outranking parameters and require an intense support by a DA. To facilitate this process, the pair DM-DA can use the Preference Disaggregation Analysis (PDA) paradigm (e.g. [61]), which has received increasing interest from the MCDA community. PDA infers the model's parameters from holistic judgments provided by the DM. Those judgments may be obtained from decisions made for a limited set of fictitious portfolios, or decisions taken for a subset of the portfolios under consideration for which the DM can easily make a judgment. In the framework of outranking methods, PDA has been recently approached in [62,63].

Fernandez et al. in [10] solved problems of allocating public funds via their outranking model. However, that work does not consider interactions among projects, which is an important concern in most practical applications.

In light of this feedback, we propose here a portfolio optimization metaheuristic approach based on the preferential model proposed in [15]. So, our metaheuristic inherits all the advantages of this model, but we have incorporated the capacity to solve portfolios with interdependent projects. Several papers in the literature consider synergy as an inherent characteristic of the portfolio problem (e.g. $[5,16,17,18,19])$. Our solution approach, called Non-Outranked Ant Colony Optimization shows promising results compared to other related algorithms. Experimental results provide evidence that it is very capable of getting close to the Pareto frontier when the best compromise is sought.

\section{Our proposal}

Our algorithm, NO-ACO (Non-Outranked Ant Colony Optimization), is based on the optimization idea proposed in [64] by Dorigo and Gambardella, which has been adapted more than once to find a set of Pareto solutions (e.g. $[16,65,66,67])$, but incorporates the preference model from [15]. The algorithm performs the optimization process through a set of agents called ants. Each ant in the colony builds a portfolio by selecting a project at a time. The way of choosing each project is called a selection rule. When all ants have finished constructing their portfolios, these are evaluated and each ant drops pheromone according to this assessment. Pheromone is used for learning, allowing the next generation of ants to acquire knowledge about the structure of the best solutions. To prevent premature convergence, the colony includes a strategic oblivion mechanism, known as evaporation, which reduces the pheromone trail over specified periods of time. In order to improve the intensification, NO-ACO includes a variable neighbourhood search for the best solutions. This local search runs once per iteration. This intensifier scheme is complemented by a diversifier mechanism, in which portfolios that have remained non-strictly-outranked for more than $\gamma$ generations are removed from the solution set. This allows the selective pressure to be relaxed. This behaviour is desirable when the algorithm has only found out local optima. The optimization process ends when a predetermined termination criterion (such as a maximum number of iterations, or a subsequent recurrence of the best solution) is reached. The following sections describe the elements of the NO-ACO algorithm in further detail.

\subsection{Pheromone representation}

Pheromone is usually represented by the Greek letter $\tau$ and is modelled in NO-ACO as a two dimensional array of size $N \times N$, where $N$ is the total number of applicant project proposals. The pheromone between two projects $i$ and $j$ is represented as $\tau_{i, j}$, and indicates how good it is that both projects receive financial support. Pheromone values are in range $(0,1]$, initializing at the upper limit to prevent premature convergence. The pheromone matrix acts as a reinforcement learning structure reflecting the knowledge gained by the ants that formed high-quality portfolios. 
The pheromone representation of NO-ACO allows identifying pairs, trios, quartets or larger project subgroups present in the best portfolios. Most likely, some synergies (mainly those that decrease costs and/or increase objectives) occur in the best portfolios. These favourable project interactions are detected through the pheromone matrix and this knowledge is transmitted to ants of the next generation for building better solutions.

\subsection{Selection rule}

Each ant builds its portfolio by selecting the projects one by one, taking into account two factors:

-Local knowledge (heuristic): This considers the benefits provided by the project to the portfolio and how many resources the project consumes. Local knowledge for the $j$ th project is denoted by $\eta_{j}$ and is calculated by the expression:

$$
\eta_{j}=\frac{\frac{1}{c(j)} \sum_{k=1}^{p} f_{k}(j)}{\max _{l \in X}\left\{\frac{1}{c(l)} \sum_{k=1}^{p} f_{k}(l)\right\}},
$$

where $c(j)$ is a measure proportional to the cost of project $j, p$ is the number of objectives, $X$ is the applicant project list, and $f_{k}(j)$ is the benefit from project $j$ to the $k$ th objective. Equation (10) promotes the inclusion of projects that have a good balance between intended objectives and requested budget. In Equation (10), $c(j)$ is defined as

$$
c(j)=\frac{1}{q} \sum_{k=1}^{q}\left(\frac{c_{j, k}}{\mathbb{B}_{k}}\right),
$$

where, $q$ is the number of categories of resources, $c_{j, k}$ is the $k$ th resource cost requested by project $j$, and $\mathbb{B}_{k}$ is the available amount of resource in the $k$ th category. -Global knowledge (learning): This takes into account the experience of previous generations of ants, expressed in the pheromone matrix. The global knowledge for project $i$ to be included in a portfolio $x$ is denoted by $\overline{\tau(x, i)}$ and is defined by the expression:

$$
\overline{\tau(x, i)}=\frac{\sum_{j=1}^{N}\left(x_{j}\right) \tau_{i, j}}{\sum_{j=1}^{N} x_{j}},
$$

where $N$ is the total number of applicant projects, $x_{j}$ is the binary value indicating whether the $j$ th project is included in the portfolio $x$, and $\tau_{i, j}$ is the pheromone for projects $i$ and $j$. The numerator in
Equation (12) is the total sum of pheromone between $i$ and each project in portfolio $x$; the denominator is the cardinality of $x$. The global knowledge favours the selection of projects that were part of the best portfolios in previous generations. At the first iteration this knowledge has no effect on portfolio formation process.

Both knowledge factors are linearly combined into a single evaluation function, which corresponds to Equation (13):

$$
\Omega(x, i)=w \cdot \eta_{i}+(1-w) \cdot \overline{\tau(x, i)},
$$

where $w$ is a parameter weighing global and local knowledge. Each ant in the colony has a different value for $w$, which is generated at random in the range $[0, W]$ with $W<1$. $W$ determines the possible greatest value of $w$ for each ant. The function $\Omega$ forms the basis of the selection rule.

If $x$ is a partially-constructed portfolio, one or more projects may be included in $x$. From among all the project proposals, only those that are not part of $x$ and the inclusion of which favours the fulfilment of budgetary constraints should be considered. This set is known as the candidate project list and is denoted by $X^{\ominus}$. Note that $X^{\ominus}$ is a subset of $X$. The choice of which $j \in X^{\ominus}$ will be added is made by using the selection rule:

$$
j= \begin{cases}\arg \max _{i \in X}\{\Omega(x, i)\} & \text { if } \wp \leq \alpha_{1}, \\ \mathcal{L}_{i \in X^{\ominus}}\{\Omega(x, i)\} & \text { if } \alpha_{1}<\wp \leq \alpha_{2}, \\ \ell_{i \in X^{\ominus}} & \text { otherwise, }\end{cases}
$$

where $j$ is the next project to be included; $\wp$ is a pseudorandom number between zero and one; $\alpha_{1}$ is a parameter that sets the intensification probability in the algorithm (choosing the project with the greatest value of $\Omega$ ); and $\alpha_{2}-\alpha_{1}$ is the probability of triggering a middle state between intensification and diversification (randomly selecting a project $i$ with probability proportional to its assessment $\Omega$ ), this selection scheme is represented by $\mathcal{L}$; in the event that $\wp>\alpha_{2}$, diversification is promoted by means of the function $\ell$ (taking a project uniformly at random).

\subsection{Pheromone laying and evaporation}

At the beginning of the first iteration, the pheromone matrix is initialized to $\tau_{i, j}=1$ for all $(i, j) \in N \times N$. After that, each ant constructs a feasible portfolio. In a colony with $n$ ants, $n$ new solutions are generated after each iteration, and there is also a set of size $m$ with the best portfolios found from the previous iterations. If all alternatives are integrated into a set $O$ whose cardinality is $n+m$, we can identify the non-strictly-outranked front $N S(O)$.

In addition, $N S(O)$ is subdivided into domination fronts. The fronts are obtained by considering the minimization of two objectives, $W(O, x)$ and $F(O, x)$, 
according to the best-compromise definition given in Problem (9). The set composed by these fronts is denoted by $\mathcal{F}=\left\{\mathcal{F}_{1}, \mathcal{F}_{2}, \ldots, \mathcal{F}_{k}, \mathcal{F}_{k+1}, \ldots\right\}$, where $\mathcal{F}_{1}$ contains the non-dominated solutions, $\mathcal{F}_{2}$ contains the portfolios that are dominated by only one solution, $\mathcal{F}_{3}$ those dominated by two solutions, and so forth. In general, the portfolios dominated by $k$ solutions are in $\mathcal{F}_{k+1}$. The set $\mathcal{F}$ will be used in the pheromone intensification in order to increase the selective pressure towards the best compromise.

Each pair of projects $(i, j)$ for each solution $x \in O$ intensifies the pheromone trail according to the expression:

$$
\tau_{i, j}= \begin{cases}\tau_{i, j}+\Delta \tau_{i, j} & \text { if } x \in N S(O) \\ \tau_{i, j} & \text { otherwise. }\end{cases}
$$

If $x$ is a non-strictly-outranked solution, then there is a $k$ such that $x \in \mathcal{F}_{k}$. The pheromone increase depends on $k$, and is defined as:

$$
\Delta \tau_{i, j}=\left(\frac{|\mathcal{F}|-k+1}{|\mathcal{F}|}\right)\left(1-\tau_{i, j}\right) \quad \text { if } x \in \mathcal{F}_{k},
$$

where $i$ and $j$ belong to portfolio $x$.

At the end of each iteration, the entire pheromone matrix is evaporated by multiplication by a constant factor lying between zero and one, denoted as $\rho$.

\subsection{Local search}

The algorithm intensification is promoted by a greedy variable-neighbourhood local search that is only carried out on non-strictly-outranked solutions. This search explores regions near to the best known solutions by a simple scheme consisting of randomly selecting $v$ projects, and generating all possible combinations of them for each solution in the non-strictly-outranked frontier. Small values for $v$ provoke behaviour that is too greedy, whereas large values produce intolerable computation times. In our experiments we obtained a good balance between these by using $v=\lceil\ln N\rceil$. The algorithmic outline for the local search is illustrated by Algorithm 1.

As observed in Algorithm 1, the search starts by choosing $v$ projects at random (Line 2), and generating all combinations of them (Line 3). Every combination is set for each portfolio in $N S(O)$ (Lines 4-11).

In Line 12, procedure repair has two main goals: 1) improving clearly-suboptimal portfolios, and 2) bringing unfeasible portfolios to the feasible region. Thus, it has two conditions to check:

-If the generated solution is partially constructed: then repair adds projects to portfolio, according to selection rule but respecting the bits assigned by the current combination (represented by $c$ in Algorithm 1). This is done until no project can be added to the portfolio.

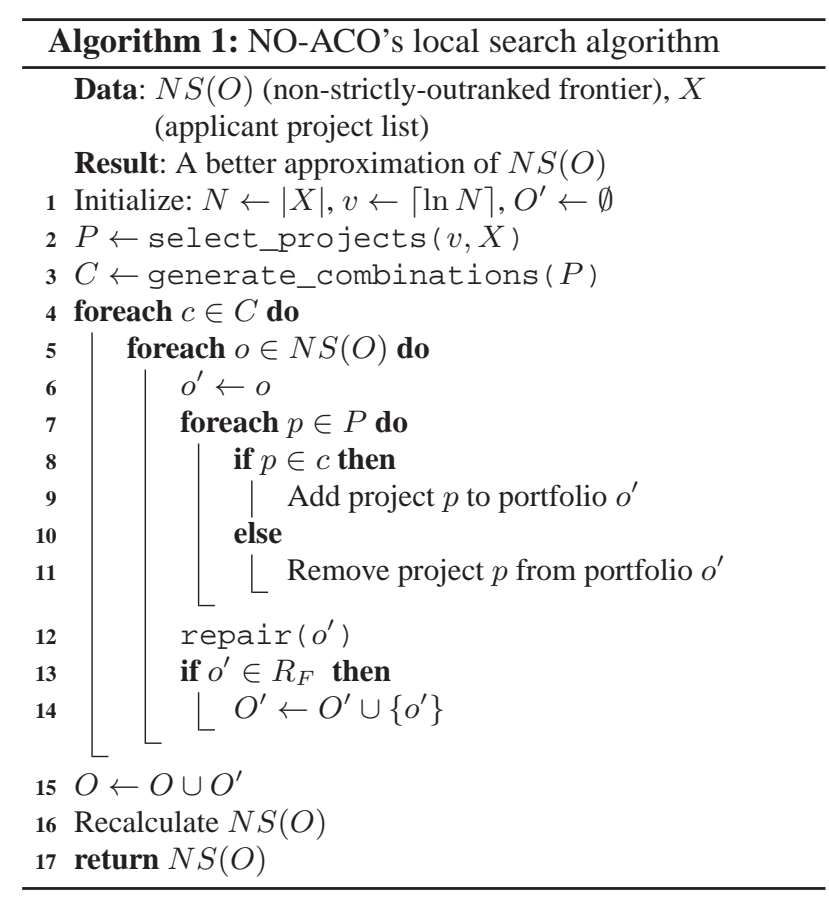

-If the generated solution is unfeasible: then repair removes projects at random until the portfolio does not surpass the budget. The probability of removing a project is inversely proportional to its expected benefits. No project chosen by the current combination can be removed. In the generated instances, repair procedure could make feasible the most of solutions.

Each feasible solution is evaluated to verify whether or not it is a non-strictly-outranked solution (Lines 13-17).

\subsection{Algorithmic description of NO-ACO}

Algorithm 2 presents an algorithmic outline of NO-ACO. Line 1 indicates the initialization of the control variables, and Lines 2-27 show the search process.

Lines 4-12 of Algorithm 2 illustrate the process of the formation of portfolios. Each ant starts from an empty portfolio, and projects are added by the selection rule, one at a time. Complete and feasible solutions are stored in $O$. These are then evaluated according to Problem (9), and the non-strictly-outranked solutions are refined by local search. Pheromone increase is the next step (Lines 14-17).

In Lines 18-23, the non-strictly-outranked set and some algorithm control variables are updated. Subsequently, at Line 24, the procedure remove_and_refill counts the number of iterations of each solution in the local $N S$ frontier. All solutions with more than $\gamma$ iterations are removed from the local set, and replaced by new solutions in the global $N S$ 


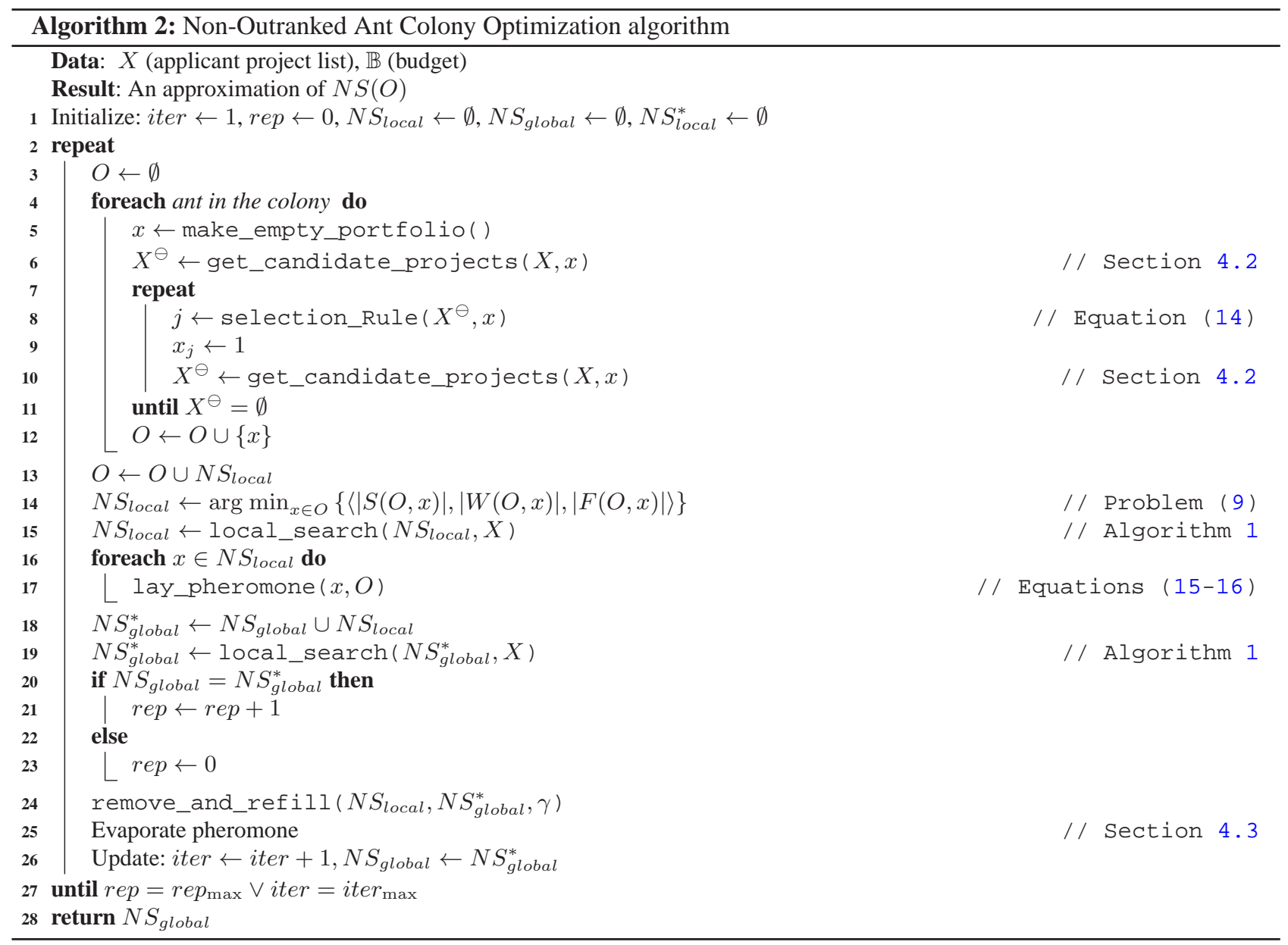

frontier. These new solutions should not have belonged to $N S_{\text {local }}$, therefore they have to be generated by the local search on $N S_{\text {global }}^{*}$. While this search is providing non-strictly-outranked portfolios the replacement will be possible. The removed solutions can still belong to the global non-strictly-outranked front, but no longer influence the optimization process made by the colony.

At the end of each iteration, pheromone is evaporated (Line 25), and the remaining algorithm control variables are updated (Line 26). The algorithm finishes when it has iterated with the same set of solutions as the non-strictly-outranked frontier during $r e p_{\max }$ iterations, or if it has reached the maximum number of iterations iter $_{\max }$ (Line 27).

\section{Case study: Optimization of social assistance portfolios}

Consider a DM facing a portfolio problem, with 100 project proposals are aimed at benefitting the most precarious social classes. The project quality is measured as the number of beneficiaries for each of nine criteria that have previously been established. Each objective is associated with one of three classes (extreme poverty, lower class and lower-middle class) and one of three levels of impact (low, medium and high).

The total budget to distribute is 250 million dollars. The proposals can be grouped into three types according to their nature, and into two geographic regions according to the location of their impact. Furthermore, in a desire to provide equitable conditions, the DM imposes the following restrictions:

1.The budget allocated to support each project type should be between $20 \%$ and $60 \%$ of the total budget.

2.The financial support allocated to each region must be at least $30 \%$ of the total budget, and no more than $70 \%$.

The DM has also identified 20 relevant interactions among projects: four of them are cannibalization phenomena, six correspond to situations of mutually-excluding projects, and ten are synergism interactions. There are up to five projects per interaction.

In order to make easier the comparative descriptions, in this section the term Pareto efficiency (and all the related terms, such as optimal or efficient portfolio) will be used to refer to non-dominated solutions of (1), and the term 
Table 1: Effect of preferences incorporation on the Pareto Ant Colony Optimization algorithm

\begin{tabular}{|c|c|c|c|c|c|c|}
\hline $\begin{array}{l}\stackrel{0}{\Xi} \\
\vdots \\
\vdots \\
\vdots\end{array}$ & Algorithm & $\begin{array}{c}\text { Time } \\
\text { (seconds) }\end{array}$ & $\begin{array}{c}\text { Size } \\
\text { of the } \\
\text { solution } \\
\text { set }\end{array}$ & $\begin{array}{c}\text { Non-dominated } \\
\text { solutions } \\
\text { in } \\
O_{1} \cup O_{2}\end{array}$ & $\begin{array}{c}\text { Solutions } \\
\text { belonging } \\
\text { to } \\
N S\left(O_{1} \cup O_{2}\right)\end{array}$ & $\begin{array}{c}\text { Obtains } \\
\text { the best } \\
\text { compromise } \\
\text { in } \mathrm{O}_{1} \cup \mathrm{O}_{2}\end{array}$ \\
\hline \multirow{2}{*}{1} & $\mathrm{P}-\mathrm{ACO}$ & 3448.07 & 2006 & 928 & 10 & \\
\hline & P-ACO-P & 536.66 & 15 & 15 & 10 & $\checkmark$ \\
\hline \multirow{2}{*}{2} & $\mathrm{P}-\mathrm{ACO}$ & 3470.29 & 2514 & 1295 & 7 & \\
\hline & P-ACO-P & 775.94 & 19 & 19 & 13 & $\checkmark$ \\
\hline \multirow{2}{*}{3} & $\mathrm{P}-\mathrm{ACO}$ & 3485.16 & 2456 & 280 & 13 & \\
\hline & P-ACO-P & 1112.49 & 34 & 34 & 17 & $\checkmark$ \\
\hline \multirow{2}{*}{4} & $\mathrm{P}-\mathrm{ACO}$ & 3591.27 & 2587 & 1392 & 10 & $\checkmark$ \\
\hline & P-ACO-P & 734.58 & 38 & 37 & 19 & $\checkmark$ \\
\hline \multirow{2}{*}{5} & $\mathrm{P}-\mathrm{ACO}$ & 3525.85 & 2245 & 1165 & 10 & \\
\hline & P-ACO-P & 1035.85 & 21 & 21 & 15 & $\checkmark$ \\
\hline \multirow{2}{*}{6} & P-ACO & 3496.68 & 2013 & 161 & 11 & \\
\hline & P-ACO-P & 855.68 & 18 & 18 & 10 & $\checkmark$ \\
\hline \multirow{2}{*}{7} & $\mathrm{P}-\mathrm{ACO}$ & 3549.55 & 2211 & 766 & 13 & $\checkmark$ \\
\hline & P-ACO-P & 161.02 & 19 & 19 & 14 & $\checkmark$ \\
\hline \multirow{2}{*}{8} & $\mathrm{P}-\mathrm{ACO}$ & 3464.27 & 2285 & 1317 & 13 & \\
\hline & P-ACO-P & 1646.32 & 28 & 28 & 21 & $\checkmark$ \\
\hline \multirow{2}{*}{9} & $\mathrm{P}-\mathrm{ACO}$ & 3707.65 & 965 & 762 & 4 & $\checkmark$ \\
\hline & P-ACO-P & 712.24 & 25 & 25 & 11 & $\checkmark$ \\
\hline \multirow{2}{*}{10} & P-ACO & 3549.67 & 2255 & 1403 & 15 & $\checkmark$ \\
\hline & P-ACO-P & 651.43 & 18 & 18 & 16 & $\checkmark$ \\
\hline
\end{tabular}

Note: $\mathrm{O}_{1}$ and $\mathrm{O}_{2}$ are the solution sets generated by P-ACO and P-ACO-P respectively.

The best compromise is the best solution to Problem (9) on $O_{1} \cup O_{2}$.

best compromise to best solutions to (9) (the best portfolio compatible with the fuzzy outranking relation $[10,15])$.

Below, we present a range of experiments to verify the validity and advantages of our approach to solving this case study. They give evidence of the benefits of incorporating the DM's preferences during the optimization process, and thus they also prove that our approach has good potential for solving real resource-allocation problems.

\subsection{Effect of incorporating the DM's preferences}

To the best of our knowledge, the P-ACO algorithm [16] is the most relevant ant colony algorithm applied to solve project portfolio selection. In order to appraise the effect of incorporating the DM's preferences on a multi-objective optimization algorithm, we implemented a version of $\mathrm{P}-\mathrm{ACO}$ that included the preferential model described in Section 3.2. This adaptation was called $\mathrm{P}-\mathrm{ACO}$ with preferences (P-ACO-P). Instead of approximating the Pareto frontier defined by the nine maximizing objectives of the problem, it searches for the best compromise expressed by Problem (9). In order to reflect a credible decision situation, we assign the values suggested by Fernandez et al. in [15] to the preferential model parameters. There is no other difference between
$\mathrm{P}-\mathrm{ACO}$ and $\mathrm{P}-\mathrm{ACO}-\mathrm{P}$. Both algorithms were programmed in Java language, using the JDK 1.6 compiler, and NetBeans 6.9.1 as Integrated Development Environment (IDE). The experiments were run on a Mac Pro with an Intel Quad-Core $2.8 \mathrm{GHz}$ processor and $3 \mathrm{~GB}$ of RAM.

The P-ACO parameter setting was that suggested in [16] by Doerner et al. The version that incorporates preferences has the same setting values.

Table 1 shows the experimental results on ten artificial instances following the case-study features.

The best compromise has been identified from solutions sets generated by both optimization methods. In this sense, that best compromise is related to the known solution set; therefore, it will be called the known best compromise, which approximates the true best compromise. This is a non-dominated solution of Problem (9) on the original objective space. So, the true best compromise must belong to the true efficient set, and it should not be strictly outranked by any other Pareto solution.

As can be seen from Table 1, incorporating preferences provides a closer approximation to a privileged region of the Pareto frontier. The version considering preferences provides solutions that dominated the $54 \%$, on average, of solutions produced by the original version of the algorithm. Probably, with many objectives, P-ACO is sensitive to the existence of dominant resistant solutions. There is also a significant 
Table 2: Efficiency analysis of NO-ACO

\begin{tabular}{|c|c|c|c|c|c|c|}
\hline $\begin{array}{l}\tilde{\Xi} \\
\vdots \\
\vdots \\
\vdots \\
\vdots\end{array}$ & Algorithm & $\begin{array}{c}\text { Time } \\
\text { (seconds) }\end{array}$ & $\begin{array}{c}\text { Size } \\
\text { of the } \\
\text { solution } \\
\text { set }\end{array}$ & $\begin{array}{c}\text { Non-dominated } \\
\text { solutions } \\
\text { in } \\
O_{1} \cup O_{2}\end{array}$ & $\begin{array}{c}\text { Solutions } \\
\text { belonging } \\
\text { to } \\
N S\left(O_{1} \cup O_{2}\right)\end{array}$ & $\begin{array}{l}\text { Obtains } \\
\text { the best } \\
\text { compromise } \\
\text { in } O_{1} \cup O_{2}\end{array}$ \\
\hline \multirow{2}{*}{1} & SS-PPS & 37946.70 & 4997 & 4981 & 12 & \\
\hline & NO-ACO & 5101.78 & 16 & 16 & 15 & $\checkmark$ \\
\hline \multirow{2}{*}{2} & SS-PPS & 23223.68 & 4996 & 4956 & 10 & \\
\hline & $\mathrm{NO}-\mathrm{ACO}$ & 2130.98 & 18 & 18 & 18 & $\checkmark$ \\
\hline \multirow{2}{*}{3} & SS-PPS & 33265.31 & 4996 & 4970 & 21 & \\
\hline & NO-ACO & 3091.89 & 29 & 29 & 28 & $\checkmark$ \\
\hline \multirow{2}{*}{4} & SS-PPS & 49865.11 & 4997 & 4946 & 24 & \\
\hline & $\mathrm{NO}-\mathrm{ACO}$ & 4720.02 & 43 & 43 & 40 & $\checkmark$ \\
\hline \multirow{2}{*}{5} & SS-PPS & 30218.23 & 4996 & 4959 & 12 & \\
\hline & NO-ACO & 4009.47 & 32 & 32 & 32 & $\checkmark$ \\
\hline \multirow{2}{*}{6} & SS-PPS & 43253.64 & 4996 & 4949 & 18 & $\checkmark$ \\
\hline & $\mathrm{NO}-\mathrm{ACO}$ & 2743.55 & 26 & 26 & 22 & $\checkmark$ \\
\hline \multirow{2}{*}{7} & SS-PPS & 29386.18 & 4973 & 4973 & 14 & \\
\hline & NO-ACO & 4512.12 & 21 & 21 & 21 & $\checkmark$ \\
\hline \multirow{2}{*}{8} & SS-PPS & 38585.35 & 4996 & 4940 & 27 & \\
\hline & $\mathrm{NO}-\mathrm{ACO}$ & 3901.76 & 35 & 35 & 35 & $\checkmark$ \\
\hline \multirow{2}{*}{9} & SS-PPS & 35514.66 & 4996 & 4936 & 9 & \\
\hline & $\mathrm{NO}-\mathrm{ACO}$ & 1238.33 & 16 & 16 & 12 & $\checkmark$ \\
\hline \multirow{2}{*}{10} & SS-PPS & 46241.69 & 4996 & 4956 & 16 & \\
\hline & $\mathrm{NO}-\mathrm{ACO}$ & 1467.29 & 20 & 20 & 20 & $\checkmark$ \\
\hline
\end{tabular}

Note: $O_{1}$ and $O_{2}$ are the solution sets generated by SS-PPS and NO-ACO respectively.

The best compromise is the best solution to Problem (9) on $O_{1} \cup O_{2}$.

run-time reduction (in the test cases, this reduction was $76 \%$ on average). Also, if the model of preferences matches with the DM's preferences, the best compromise among the set of all portfolios generated is always identified by P-ACO-P. Furthermore, when the DM has to choose one alternative as the final decision, the thousands of portfolios from $\mathrm{P}-\mathrm{ACO}$ make it difficult to reach a decision. By incorporating preferences, this drawback is very strongly reduced.

\subsection{Evaluation of NO-ACO solutions}

For the problem presented in this section, the only way to ensure that a solution is the true best compromise is if we know the whole true Pareto frontier, or at least, the full non-strictly-outranked frontier. For instances of large size like those we have addressed, it is not possible to know with certainty the Pareto frontier. However, there are methods reported in the literature that can approximate this frontier with an acceptable error.

In order to verify whether the NO-ACO solutions acceptably approximate the true Pareto frontier, we have estimated the Pareto set by means of SS-PPS, as proposed by Carazo et al. $[18,19]$. This is one of the most recent algorithms for portfolio optimization, and experimental tests prove its high performance, outperforming SPEA2. SS-PPS solved the case-study instances by finding a representative sample of up to five thousand efficient points according to the parameter setting suggested in $[18,19]$.

NO-ACO was programmed in Java language, using the JDK 1.6 compiler, and NetBeans 6.9.1 as IDE. The experiments were run on a Mac Pro with an Intel Quad-Core 2.8 GHz processor and $3 \mathrm{~GB}$ of RAM.

Again we used the values suggested in [15] for the preferential model parameters. Besides, the NO-ACO parameter setting used to obtain the results in this section is: $\alpha_{1}=0.65, \alpha_{2}=0.85, \rho=0.9, \gamma=25, W=0.60$, $r e p_{\max }=50$ and iter $_{\max }=100000$. Moreover, the colony has one hundred ants. This setting was obtained from exploring parameter values with the objective of achieving a good algorithmic performance. Taking into account the results in a wide range of instances, we consider that these parameter values are robust enough to maintain an efficient behavior of NO-ACO.

We want to give evidence that our approach acceptably approximates the best compromise. With this aim, we solved the same ten instances from Section 5.1. For these, we have approximated: 1) the best compromise by using NO-ACO, and 2) the Pareto frontier by means of SS-PPS.

The results are summarized in Table 2. On analysing the data, we may conclude that our algorithm has efficient behaviour. NO-ACO got close to the Pareto frontier better than SS-PPS in the most preferred region (the so-called $\mathrm{RoI}$ ), that is, the non-strictly-outranked frontier. No 
Table 3: A sample of the non-strictly-outranked frontier generated by NO-ACO compared to the ranking-based solution

\begin{tabular}{|c|c|c|c|c|c|c|c|c|c|c|c|c|c|c|c|c|c|c|c|c|}
\hline & \multirow{2}{*}{ 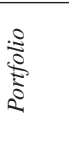 } & \multicolumn{16}{|c|}{$\begin{array}{c}\text { Values of } \\
\text { objective functions }\end{array}$} & \multicolumn{3}{|c|}{$\begin{array}{l}\text { Number of solutions } \\
\text { that outranks it }\end{array}$} \\
\hline & & 1 & 2 & 3 & 4 & 5 & 6 & 7 & 8 & 9 & 10 & 11 & 12 & 13 & 14 & 15 & 16 & strictly & weakly & $\begin{array}{l}\text { in net flow } \\
\text { score }\end{array}$ \\
\hline \multirow{9}{*}{ 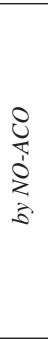 } & 1 & 106 & 806 & 504 & 612 & 107 & 811 & 502 & 605 & 983 & 871 & 473 & 610 & 108 & 847 & 499 & 597 & 0 & 0 & 0 \\
\hline & 2 & 96 & 766 & 467 & 556 & 98 & 786 & 459 & 562 & 988 & 772 & 457 & 565 & 98 & 756 & 454 & 545 & 0 & 0 & 1 \\
\hline & 3 & 98 & 730 & 461 & 562 & 99 & 740 & 475 & 564 & 988 & 796 & 464 & 563 & 95 & 767 & 453 & 541 & 0 & 1 & 2 \\
\hline & 4 & 100 & 742 & 479 & 545 & 94 & 744 & 459 & 565 & 992 & 785 & 451 & 547 & 96 & 745 & 447 & 535 & 0 & 2 & 1 \\
\hline & 5 & 96 & 742 & 462 & 553 & 95 & 751 & 456 & 562 & 999 & 809 & 454 & 562 & 94 & 776 & 452 & 546 & 0 & 2 & 1 \\
\hline & 6 & 98 & 743 & 462 & 550 & 95 & 730 & 473 & 559 & 991 & 765 & 460 & 553 & 95 & 740 & 450 & 541 & 0 & 2 & 5 \\
\hline & 7 & 98 & 746 & 466 & 556 & 98 & 769 & 454 & 569 & 990 & 790 & 447 & 565 & 94 & 770 & 454 & 547 & 0 & 3 & 3 \\
\hline & 8 & 92 & 739 & 469 & 557 & 91 & 753 & 445 & 556 & 990 & 784 & 468 & 565 & 90 & 738 & 440 & 549 & 0 & 4 & 6 \\
\hline & 9 & 98 & 733 & 461 & 556 & 95 & 750 & 448 & 567 & 987 & 791 & 454 & 565 & 97 & 777 & 454 & 556 & 0 & 8 & 7 \\
\hline \multicolumn{2}{|c|}{ ranking-based } & 96 & 736 & 471 & 558 & 95 & 762 & 453 & 561 & 944 & 768 & 469 & 565 & 97 & 756 & 436 & 540 & 9 & 0 & 9 \\
\hline
\end{tabular}

NO-ACO solution is dominated by an SS-PPS one, and our approach could dominate 16-60 solutions suggested by the other method. Additionally, our proposal was able to identify the best compromise from the entire approximated frontier, using only, on average, $10 \%$ of the time required to estimate the whole Pareto set.

There is evidence of the advantages of incorporating the DM's preferences: it decreases the computational effort and increases the algorithm efficiency on the solution region that best matches the DM's formulated preferences.

In Table 2, the best compromises are related to the outranking model's parameters that were set a priori. In multi-objective optimization, the DM 'learns' trade-offs while he/she finds and judges new Pareto solutions; thus his/her aprioristic preferences could be modified. Once the best compromise and others non-strictly outranked solutions have been obtained and evaluated by the DM, the model's parameter setting may be updated, perhaps using PDA as proposed in [63]. If the parameter values were modified, with an additional NO-ACO run the final best compromise should be reached.

\subsection{Solving problems with high dimensionality}

The test in the previous section was limited to 100 projects and nine objectives. These dimensions exceed those addressed by most studies in the specialized literature (e.g. [5,16,17,18, 19,68]). These dimensions are appropriate for most portfolio problems in the business sector; however, in public organizations, the problem size may be larger. In order to explore the capacity of our algorithm to solve instances with a large size, we generated a set of instances with 500 projects and 16 criteria to optimize.

The interpretation is similar to that described at the beginning of this section: there is a budget of 250 million dollars to distribute, and the DM wants to keep a balance so has grouped the projects into two areas and three regions and imposed budgetary constraints for each (30\%-70\% for each area and $20 \%-60 \%$ for each region).

In addition, the DM has identified 100 relevant interactions between projects: 20 are cannibalization phenomena, 30 correspond to redundant projects and 50 are synergies that generate added value.

Unlike the 100-projects case, it is not possible in these instances to generate an acceptable approximation of the Pareto frontier that can be used as reference for comparison purposes. Even the best multi-objective algorithms are degraded when they attempt to generate it. This is combined with computation times that would be intolerable or with an abrupt interruption of the algorithms if they fail to converge towards the frontier.

In order to test the quality of the solutions suggested by our proposal, a comparison with a popular acceptable way of allocating resources can be performed. Among several heuristics frequently used, we chose one based on assigning budgetary resources according to project-ranking information. Here, a project ranking is built by using a cost-benefit ratio; the benefit is modelled by a weighted sum, whose weights are adjusted to reflect the DM's preferences. The project ranking is built following the order given by the cost-benefit ratio. Once the set of projects has been ranked, the resources may be allocated by following the priorities implicit in the rank order until no resources are left. This at least ensures the inclusion of projects that provide more benefit per dollar. Synergism can be tackled if the project interactions are modelled as dummy projects that can also be ranked.

Table 3 concentrates on only nine of the 164 solutions found by NO-ACO as an approximation to the non-strictly-outranked frontier. Our algorithm converges after 41,625 seconds. The best compromise that was found (Solution 1) outperforms the ranking-based portfolio, even in the Pareto sense.

Another ten instances were generated following the same features. When they were solved by NO-ACO, we observed the same behaviour: the ranking-based portfolio was dominated by the best compromise found by 
NO-ACO. This test gives some evidence of the applicability of our approach to large-scale real instances.

\section{Conclusions and future work}

We have presented an original proposal to optimize interdependent projects portfolios. This proposal is an adaptation of the well-known ant colony optimization metaheuristic, but incorporates preferences based on the outranking model of Fernandez et al. [10]. Our algorithm (NO-ACO) searches for optimal portfolios in synergetic conditions and can handle interactions impacting both objectives and costs. Redundancy is also considered during portfolio formation. By incorporating preferences, the selective pressure toward a privileged zone of the Pareto frontier is increased. Thus, a zone that matches the DM's preferences better can be identified. In comparison with other metaheuristic approach that does not incorporate preferences, NO-ACO achieves greater closeness to the region of interest with less computational effort. Our result seems to confirm the hypothesis from $[10,15]$ : the incorporation of DM preferences by solving Problem (9) helps to obtain solutions that dominate others from leading metaheuristics.

Since it is enriched by preferences, our proposal acquires the ability to find good solutions (the known best portfolio) to portfolio problems with higher dimensions (in project and objective spaces) than those reported in scientific literature. Compared to the popular ranking-based method, NO-ACO finds solutions that outperform to the ranking-based portfolio, both in Pareto dominance and in strict outranking.

As immediate work we are going to explore the limits of this approach, by finding the greatest size of the instances that can be solved with an acceptable performance. Additionally, we are going to incorporate an interactive process for updating the preference model according to the new information gained by the DM from the optimized solutions.

\section{Acknowledgements}

We acknowledge the support from PROMEP through the network project 'Optimization and Decision Support'. We also thank to DGEST and the Autonomous University of Sinaloa (PROFAPI-055/2012 project).

\section{References}

[1] D N Kleinmuntz. Portfolio Decision Analysis: Improved methods for resource allocation, chapter Foreword, pages v-vii. Springer, New York-Dordrecht-Heidelberg-London, 2011.
[2] A Salo, J Keisler, and A Morton. Portfolio Decision Analysis: Improved methods for resource allocation, chapter An invitation to Portfolio Decision Analysis, pages 3-27. Springer, New York-Dordrecht-Heidelberg-London, 2011.

[3] M A Coffin and B W Taylor. Multiple criteria R\&D project selection and scheduling using fuzzy sets. Comput Oper Res, 23(3):207-220, 1996.

[4] J Klapka, P Pinos, and V Sevcik. Multicriterial projects selection. Handbook of Optimization, Intelligent Systems Reference Library, 38:245-261, 2013.

[5] C Stummer and K Heidenberger. Interactive R\&D portfolio analysis with project interdependencies and timeprofiles of multiple objectives. IEEE T Eng Manage, 50(2):175-183, 2003.

[6] J L Ringuest, S B Graves, and Case R H. Mean-gini analysis in R\&D portfolio selection. Eur J Oper Res, 154(1):157-169, 2004.

[7] C Carlsson, R Fuller, M Heikkila, and Majlender P. A dynamic and fuzzy modeling approach for multi-objective $\mathrm{R} \& \mathrm{D}$ project portfolio selection. Int J Approx Reason, 44(2):93-105, 2007.

[8] X Zhao, Y Yang, G Wu, J Yang, and X Xue. A dynamic and fuzzy modeling approach for multi-objective R\&D project portfolio selection. J Converg Inf Technol, 7(1):36-44, 2012.

[9] W Hallerbach, H Ning, A Soppe, and J Spronk. A framework for managing a portfolio of socially responsible investments. Eur J Oper Res, 153(2):517-529, 2004.

[10] E Fernandez, E Lopez, G Mazcorro, R Olmedo, and C A Coello Coello. Application of the Non-Outranked Sorting Genetic Algorithm to public project portfolio selection. Inf Sci, 228:131-149, 2013.

[11] Georgia Department of Transportation. project list and final investment report. available in http:// www.dot.ga.gov/ localgovernment/ fundingprograms/ transreferendum/ pages/projectlist.aspx (october 4th, 2012).

[12] Georgia Department of Transportation. Central Savannah River Area, unconstrained project list by county. available in http:// www.it3.ga.gov/ documents/ unconstrainedlist/ centralsavannah-unconstrainedlist.pdf (october 4th, 2012).

[13] Georgia Department of Transportation. Heart of Georgia, Altamaha unconstrained project list by county. available in http:// www.it3.ga.gov/ documents/ unconstrainedlist/ heartofgeorgia-unconstrainedlist-fullset.pdf (october 4th, 2012).

[14] Georgia Department of Transportation. River Valley Area, unconstrained project list by county. available in http:// www.it3.ga.gov/ documents/ unconstrainedlist/ rivervalleyunconstrainedlist.pdf (october 4th, 2012).

[15] E Fernandez, E Lopez, F Lopez, and C A Coello Coello. Increasing selective pressure towards the best compromise in evolutionary multiobjective optimization: The extended NOSGA method. Inf Sci, 181(1):44-56, 2011.

[16] K F Doerner, W J Gutjahr, R F Hartl, C Strauss, and C Stummer. Pareto Ant Colony optimization: A metaheuristic approach to multiobjective portfolio selection. Ann Oper Res, 131(1-4):79-99, 2004.

[17] K F Doerner, W J Gutjahr, R Hartl, C Strauss, and C Stummer. Pareto Ant Colony Optimization with ILP preprocessing in multiobjective project portfolio selection. Eur J Oper Res, 171(3):830-841, 2006. 
[18] A F Carazo, T Gomez, J Molina, A G Hernández-Díaz, F M Guerrero, and R Caballero. Solving a comprehensive model for multiobjective project portfolio selection. Comput Oper Res, 37(4):630-639, 2010.

[19] A F Carazo, I Contreras, T Gomez, and F Perez. A project portfolio selection problem in a group decisionmaking context. J Ind Manage Optim, 8(1):243-261, 2012.

[20] C L Hwang and A S M Masud. Multiple objective decision making, methods and applications: a state-of-theart survey. Lecture notes in economics and mathematical systems. Springer-Verlag, 1979.

[21] F Ghasemzadeh, N Archer, and P Iyogun. A zero-one model for project portfolio selection and scheduling. J Oper Res Soc, 50(7):745-755, 1999.

[22] B Amiri. A multi-objective hybrid optimization algorithm for project selection problem. J Basic Appl Sci Res, 2(7):6995-7002, 2012.

[23] T Kremmel, J Kubalik, and S Biffl. Software project portfolio optimization with advanced multi-objective evolutionary algorithm. Appl Soft Comput, 11(1):1416-1426, 2011.

[24] A Chen and Ch Chyu. Applying memetic algorithm in multi-objective resource allocation among competing projects. J Softw, 5(8):802-809, 2010.

[25] J Gaytán and J García. Multicriteria decision on interdependent infrastructure transportation projects using an evolutionary-based framework. Appl Soft Comput, 9(2):512526, 2009.

[26] S Ghorbani and M Rabbani. A new multi-objective algorithm for a project selection problem. Adv Eng Softw, 40(1):9-14, 2009.

[27] C M Lin and M Gen. Multicriteria human resource allocation for solving multistage combinatorial optimization problems using multiobjective hybrid genetic algorithm. Expert Syst Appl, 34(4):2480-2490, 2008.

[28] W J Gutjahr, S Katzensteiner, P Reiter, C Stummer, and M Denk. Multi-objective decision analysis for competenceoriented project portfolio selection. Eur J Oper Res, 205(3):670-679, 2010.

[29] E Zitzler, M Laumanns, and L Thiele. SPEA2: Improving the strength pareto evolutionary algorithm. Technical Report 103, Computer Engineering and Networks Laboratory (TIK), Swiss Federal Institute of Technology (ETH), 2001.

[30] H Ishibuchi, N Tsukamoto, and Y Nojima. Evolutionary many-objective optimization: A short review. In Proceedings of Evolutionary Computation, 2008 (CEC'2008), pages 2419-2426. IEEE, 2008.

[31] D W Corne and J D Knowles. Techniques for highly multiobjective optimisation: some nondominated points are better than others. In Proceedings of the 9th annual conference on Genetic and evolutionary computation, GECCO'07, pages 773-780, New York, NY, USA, 2007.

[32] J López, C A COello Coello, K Oyama, and Fujii K. Alternative preference relation to deal with manyobjective optimization problems. In R C Purshouse, P J Fleming, C M Fonseca, S Greco, and J Shaw, editors, Proceedings of Evolutionary Multi-Criterion Optimization EMO 2013, Lecture Notes in Computer Science, pages 291306. Springer-Verlag, 2013.

[33] R C Purshouse and P J Fleming. Evolutionary manyobjective optimisation: An exploratory analysis. In
Evolutionary Computation, 2003. CEC'03. The 2003 Congress on, volume 3, pages 2066-2073. IEEE, 2003.

[34] S Huband, L Barone, $P$ Hingston, While $R$ L, D Tuppurainen, and R Bearman. Designing comminution circuits with a multi-objective evolutionary algorithm. In Congress on Evolutionary Computation, pages 1815-1822, 2005.

[35] G A Miller. The magical number seven, plus or minus two: some limits on our capacity for processing information. Psychological Review, 63(2):81-97, 1956.

[36] K Deb, J Sundar, U Bhaskara, and S Chaudhuri. Reference point based multiobjective optimization using evolutionary algorithms. Int J Comput Intell Res, 2(3):273-286, 2006.

[37] S Bechikh. Incorporating Decision Maker's Preference Information in Evolutionary Multi-objective Optimization. $\mathrm{PhD}$ thesis, High Institute of Management of Tunis, University of Tunis, Tunis, Tunisia, 2013.

[38] K Deb. Multi-objective evolutionary algorithms: Introducing bias among pareto optimal solutions. Technical Report KanGAL Report 99002, Indian Institute of Technology, Kanpur, India, 1999.

[39] J Branke and K Deb. Knowledge Incorporation in Evolutionary Computation, chapter Integrating user preferences into evolutionary multi-objective optimization, pages 461-478. Springer-Verlag, Berlin-Heidelberg, 2004.

[40] E Zitzler, D Brockhoff, and L Thiele. The hypervolume indicator revisited: On the design of pareto-compliant indicators via weighted integration. In Proceedings of the 4th international conference on Evolutionary Multi-criterion Optimization (EMO'07), pages 862-876, Matsushima, Japan, 2007. Springer-Verlag.

[41] G W Greenwood, X S Hu, and J C D'ambrosio. Foundations of Genetic Algorithms, chapter Fitness functions for multiple objective optimization problems: combining preferences with Pareto rankings, pages 437-455. Morgan Kaufmann, 1996.

[42] K Deb, A Sinha, P Korhonen, and J Wallenius. An interactive evolutionary multiobjective optimization method based on progressively approximated value functions. IEEE T Evolut Comput, 14(5):723-739, 2010.

[43] M Köksalan and I Karahan. An interactive territory defining evolutionary algorithm: itdea. Evolut Comput, 14(5):702$722,2010$.

[44] R Battiti and A Passerini. Brain-computer evolutionary multiobjective optimization: A genetic algorithm adapting to the decision maker. IEEE T Evolut Comput, 14(5):671-687, 2010.

[45] J W Fowler, ES Gel, M Köksalan, P Korhonen, J L Marquis, and $\mathrm{J}$ Wallenius. Interactive evolutionary multi-objective optimization for quasi-concave preference functions. IEEE T Evolut Comput, 206(2):417-425, 2010.

[46] J Branke, S Greco, R Slowinski, and P Zielniewski. Interactive evolutionary multiobjective optimization driven by robust ordinal regression. Bull Polish Acad Sci, 58(3):347358, 2010.

[47] Y C Jin and B Sendhoff. Incorporation of fuzzy preferences into evolutionary multiobjective optimization. In Proceedings of the 4th Asia-Pacific conference on Simulated Evolution and Learning, Nanyang, Singapore, 2002.

[48] D Cvetkovic and I C Parmee. Preferences and their application in evolutionary multiobjectiveoptimisation. IEEE T Evolut Comput, 6(1):42-57, 2002. 
[49] L Rachmawati and D Srinivasan. Incorporating the notion of relative importance of objectives in evolutionary multiobjective optimization. IEEE T Evolut Comput 2010, 14(4):530-546, 2010.

[50] K Deb and A Kumar. Interactive evolutionary multiobjective optimization and decision making using reference direction method. In Proceedings of the 9th Genetic and Evolutionary Computation Conference (GECCO'07), pages 781-788, London, UK, 2007. ACM.

[51] R Allmendinger, X Li, and J Branke. Reference pointbased particle swarm optimization using a steady-state approach. In Proceedings of the 7th international conference on Simulated Evolution and Learning (SEAL'08), pages 200209, Melbourne, Australia, 2008. Springer-Verlag.

[52] J Molina, L V Santana-Quintero, A G Hernández-Díaz, C A Coello Coello, and R Caballero. g-dominance: Reference point based dominance for multiobjectivemetaheuristics. Eur J Oper Res, 197(2):685-692, 2009.

[53] S Bechikh, S L Ben, and K Ghédira. The r-dominance: a new dominance relation for preference-based evolutionary multi-objective optimization. Technical Report BS-2010001, SOIE Research Unit, High Institute of Management of Tunis, University of Tunis, Tunis, Tunisia, 2010.

[54] A López, A Arias, and C A Coello Coello. Preference incorporation to solve many-objective airfoil design problems. In Proceedings of IEEE Congress on Evolutionary Computation (CEC'11), pages 1605-1612, New Orleans, 2011. IEEE.

[55] J Branke, T Kaussler, and H Schmeck. Guidance in evolutionary multi-objective optimization. Adv Eng Softw, 32(6):499-507, 2001.

[56] E Fernandez, E Lopez, S Bernal, C A Coello Coello, and J Navarro. Evolutionary multiobjective optimization using an outranking-based dominance generalization. Comput Oper Res, 37(2):390-395, 2010.

[57] $\mathrm{T}$ Wagner and $\mathrm{H}$ Trautmann. Integration of preferences in hypervolume-based multiobjective evolutionary algorithms by means of desirability functions. IEEE T Evolut Comput, 14:688-701, 2010.

[58] B Roy. Multicriteria Methodology for Decision Aiding. Nonconvex Optimization and Its Applications. Springer, 1996.

[59] B Roy. Reading in multiple criteria decision aid, chapter The Outranking Approach and the Foundations of ELECTRE methods, pages 155-183. Springer-Verlag, 1990.

[60] J Brans and B Mareschal. Multiple Criteria Decision Analysis: State of the Art Surveys, chapter Promethee methods, pages 163-190. International Series on Operations Research \& Management Science. Springer-Verlag, Berlin, 2005.

[61] E Jacquet-Lagreze and Y Siskos. Preference disaggregation: 20 years of mcda experience. Eur J Oper Res, 130(2):233$245,2001$.

[62] M Doumpos, Y Marinakis, M Marinaki, and C Zopounidis. An evolutionary approach to construction of outranking models for multicriteria classification: The case of the ELECTRE TRI method. Eur J Oper Res, 199(2):496-505, 2009.

[63] E Fernandez, J Navarro, and G Mazcorro. Evolutionary multi-objective optimization for inferring outranking model's parameters under scarce reference information and effects of reinforced preference. Found Comput Decis Sci, 37(3):163197, 2012.

[64] M Dorigo and L M Gambardella. Ant colony system: A cooperative learning approach to the traveling salesman problem. IEEE T EvolutComput, 1(1):53-66, 1997.

[65] A Berrichi, F Yalaoui, L Amodeo, and M Mezghiche. Bi-objective ant colony optimization approach to optimize production and maintenance scheduling. Comput Oper Res, 37(9):1584-1596, 2010.

[66] S Chaharsooghi and A H M Kermani. An effective ant colony optimization algorithm for multi-objective resource allocation problem. Appl Math Comput, 200(1):167-177, 2008.

[67] B Yagmahan and M M Yenisey. Ant colony optimization for multi-objective flow shop scheduling problem. Comput Ind Eng, 54(3):411-420, 2008.

[68] J Liesiö, P Mild, and A Salo. Robust portfolio modeling with incomplete cost information and project interdependency. Eur J Oper Res, 190(3):679-695, 2008.

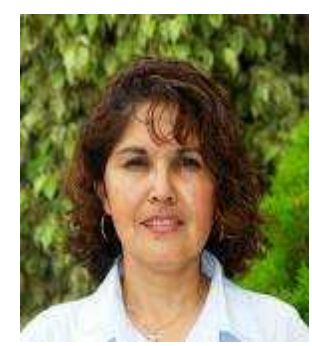

Laura Cruz received the M.Sc. degree in Computer Science from Instituto Tecnológico y de Estudios Superiores de Monterrey (Mexico) in 1999 and she received the $\mathrm{Ph} . \mathrm{D}$ (Computer Science) degree from Centro Nacional de Investigación y Desarrollo Tecnológico (Mexico) in 2004. She is a full time professor at Madero Institute of Technology, Mexico. Her research interests include optimization techniques, complex networks, autonomous agents and algorithm performance explanation. Laura Cruz is a member of the Mexican National System of Researchers and the Mexican Society of Operation Research. Professor Cruz is referee of some international journals in the frame of metaheuristic optimization.

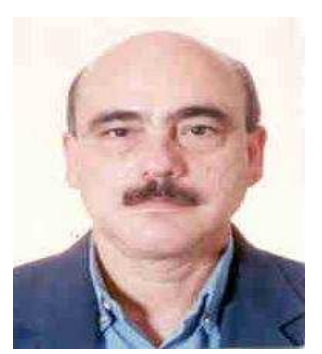

Eduardo Fernandez received the Ph.D degree in Computer Aided Design of Electronic Circuits, from Poznan University of Technology, 1987. He is currently Senior Professor of the Faculty of Civil Engineering, Autonomous University of Sinaloa (UAS),

Mexico. His main areas of interest are multi-criteria and intelligent decision support, multi-objective optimization, group decision models and project portfolio selection. In those fields he has published more than eighty papers and book chapters. Professor Fernandez is a member of the Mexican National System of Researchers, 
the Mexican Society of Operation Research, the International EUREKA Network for Knowledge Discovery, Knowledge Management and Decision Support, the International Society on Multi Criteria Decision Making and the Euro Working Group on Multi-Criteria Decision. He has been nominated three times for "OR in Development" Prize.

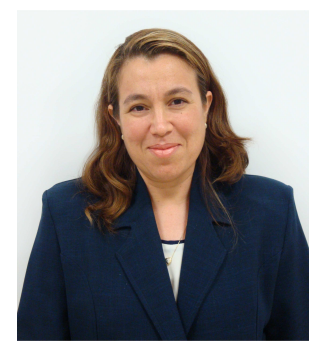

Claudia Gomez was born in Mexico in 1971. She is a full time professor at Madero Institute of Technology. She received the Ph.D degree in Advanced Technology from National Polytechnic Institute (Mexico), in 2009. She received the M.Sc. degree in Computer Science from Leon Institute of Technology (Mexico), in 2000. Her research interests are optimization techniques, complex network and autonomous agents.

Gilberto Rivera was

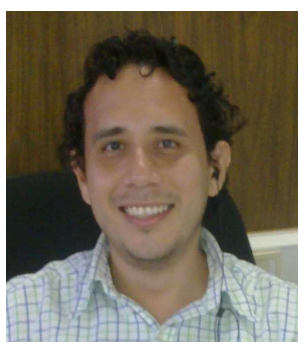
born in Mexico in 1984. He is a Ph.D student at Tijuana Institute of Technology. He received the M.Sc. degree in Computer Science from Madero Institute of Technology (Mexico), in 2010. His research interests are in the areas of project portfolio selection, optimization techniques, swarm intelligence and autonomous agents.

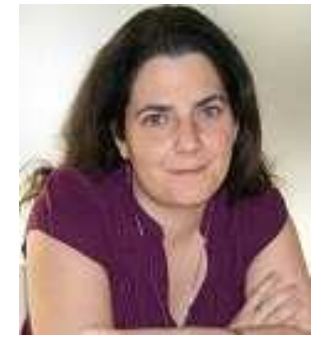

Fatima Perez is a Research Fellow at University of Malaga (Spain). She received the Ph.D degree in Mathematical Economics at University of Malaga. She has published research articles in reputed international journals in the frame of applied mathematics and applied economics. Her research interests are in the areas of project portfolio selection, multiobjective programming, metaheuristic algorithms, development of computer software and composite indicators. She is referee of some international journals in the frame of multiobjective programming. 